\title{
Experimental determination of the magnetic interactions of frustrated Cairo pentagon lattice materials
}

\author{
Manh Duc Le $\odot,{ }^{1,2,{ }^{*}}$ Elisa M. Wheeler $\odot,{ }^{3,4}$ Jaehong Jeong, ${ }^{2,5}$ K. Ramesh Kumar, ${ }^{2}$ Seongsu Lee, ${ }^{6}$ Chang-Hee Lee $\odot,{ }^{6}$ \\ Myeong Jun Oh, ${ }^{7}$ Youn-Jung Jo, ${ }^{7}$ Akihiro Kondo, ${ }^{8}$ Koichi Kindo, ${ }^{8}$ U. Stuhr $\odot,{ }^{9}$ B. Fåk, ${ }^{3}$ M. Enderle, ${ }^{3}$ Dmitry Batuk, ${ }^{10}$ \\ Artem M. Abakumov, ${ }^{11}$ Alexander A. Tsirlin, ${ }^{12}$ and Je-Geun Park ${ }^{2,5,13}$ \\ ${ }^{1}$ ISIS Neutron and Muon Source, Rutherford Appleton Laboratory, Chilton, Didcot OX11 OQX, United Kingdom \\ ${ }^{2}$ IBS Research Center for Correlated Electron Systems, Seoul National University, Seoul 08826, Korea \\ ${ }^{3}$ Institut Laue-Langevin, Avenue des Martyrs, CS 20156, F-38042 Grenoble Cédex 9, France \\ ${ }^{4}$ Cellule DATA, Université de Strasbourg, 20a rue René Descartes, F-67000 Strasbourg, France \\ ${ }^{5}$ Department of Physics and Astronomy, Seoul National University, Seoul 08826, Korea \\ ${ }^{6}$ Neutron Science Division, Korea Atomic Energy Research Institute, Daejeon 34057, Korea \\ ${ }^{7}$ Department of Physics, Kyungpook National University, Daegu 41566, Korea \\ ${ }^{8}$ Institute for Solid State Physics, The University of Tokyo, Kashiwa, Chiba 277-8581, Japan \\ ${ }^{9}$ Laboratory for Neutron Scattering, Paul-Scherrer-Institut, 5232 Villigen PSI, Switzerland \\ ${ }^{10}$ EMAT, University of Antwerp, Groenenborgerlaan 171, B-2020 Antwerp, Belgium \\ ${ }^{11}$ Skolkovo Institute of Science and Technology, Nobel Str. 3, 143026 Moscow, Russia \\ ${ }^{12}$ Experimental Physics VI, Center for Electronic Correlations and Magnetism, University of Augsburg, 86159 Augsburg, Germany \\ ${ }^{13}$ Center for Quantum Materials, Seoul National University, Seoul 08826, Korea
}

(Received 28 October 2020; revised 15 January 2021; accepted 25 February 2021; published 16 March 2021)

\begin{abstract}
We present inelastic neutron scattering measurements of the Cairo pentagon lattice magnets $\mathrm{Bi}_{2} \mathrm{Fe}_{4} \mathrm{O}_{9}$ and $\mathrm{Bi}_{4} \mathrm{Fe}_{5} \mathrm{O}_{13} \mathrm{~F}$, supported by high field magnetization measurements of $\mathrm{Bi}_{2} \mathrm{Fe}_{4} \mathrm{O}_{9}$. Using linear spin wave theory and mean-field analyses we determine the spin exchange interactions and single-ion anisotropy in these materials. The Cairo lattice is geometrically frustrated and consists of two inequivalent magnetic sites, both occupied by $\mathrm{Fe}^{3+}$ ions and connected by two competing nearest neighbor interactions. We found that one of these interactions, coupling nearest neighbor spins on the threefold symmetric sites, is extremely strong and antiferromagnetic. These strongly coupled dimers are then weakly coupled to a framework formed from spins occupying the other inequivalent site. In addition, we found that the $\mathrm{Fe}^{3+} S=5 / 2$ spins have a non-negligible single-ion anisotropy, which manifests as a spin anisotropy gap in the neutron spectrum and a spin-flop transition in high field magnetization measurements.
\end{abstract}

DOI: 10.1103/PhysRevB.103.104423

\section{INTRODUCTION}

Competing exchange interactions in geometrically frustrated magnets may lead to a large number of degenerate ground states [1]. The prototypical case is the Ising antiferromagnet on a triangular lattice, in which only half of the nearest neighbor interactions may be satisfied by any particular spin arrangement. In the more conventional Heisenberg case, where the spins are free to adopt a range of orientations, a unique ground state with spins oriented at $120^{\circ}$ to their neighbors may be stabilized. Besides triangles, other odd-vertex polygons will also exhibit geometrical frustration, however a tiling of these shapes in a regular crystal is forbidden by symmetry. Nonetheless, lattices exists which consists of tilings of irregular odd-vertex polygons, of which the Cairo pentagonal lattice is one. In this case, the pentagons have four equal and one unequal sides, as illustrated in Fig. 1(a).

This gives rise to two inequivalent sites with three- and fourfold rotational symmetry perpendicular to the pentagon

\footnotetext{
*duc.le@stfc.ac.uk
}

plane, which are connected by two competing nearest neighbor interactions. One coupling is along the four equal edges which links threefold to fourfold sites $\left(\mathcal{J}_{43}\right)$, and the other goes via the single unequal edge of the pentagon, which links two threefold sites $\left(\mathcal{J}_{33}\right)$. When these interactions are antiferromagnetic the system is geometrically frustrated and a frustration index may be constructed as $x=\mathcal{J}_{43} / \mathcal{J}_{33}$. This is small when $\mathcal{J}_{33}$ dominates, leading the nearest neighbor threefold sites (denoted $\mathrm{Fe}^{3}$ ) to form antiparallel pairs which may be thought of as dimers. Neighboring threefold pairs are connected via the fourfold sites (denoted $\mathrm{Fe}^{4}$ ) and the competing $\mathcal{J}_{43}$ interaction. If $\mathcal{J}_{43}$ is small, then for classical (Heisenberg) spins, a noncollinear spin configuration analogous to the $120^{\circ}$ structure of the triangular lattice antiferromagnets is theoretically predicted [2]. In this orthogonal structure, shown in Fig. 1(b), neighboring threefold pairs are aligned at $90^{\circ}$ to each other, as are next-nearest neighbor fourfold spins. For quantum spins, in the small $x$ limit, instead of the orthogonal structure, a collinear, partial ordered structure is predicted to be stabilized, where half of the threefold sites do not order. Finally, when $\mathcal{J}_{43}$ dominates, at large $x$, a collinear ferrimagnetic structure is predicted where the threefold and 
(a)

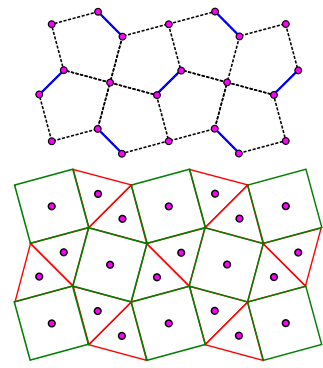

(c)

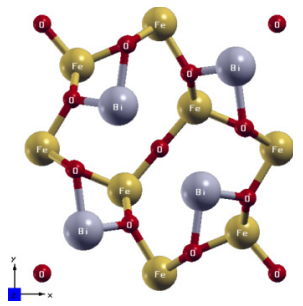

(b)

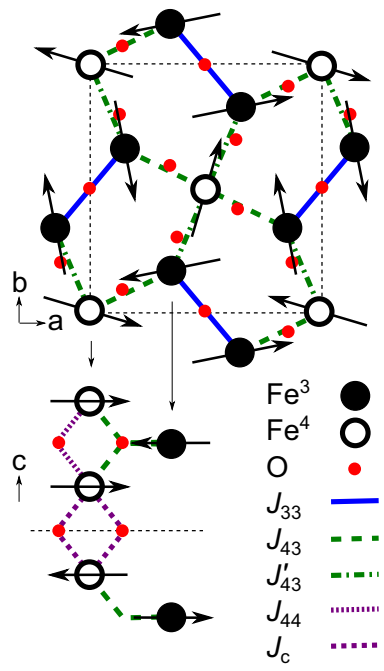

FIG. 1. (a) The Cairo lattice, with magnetic ions at the vertices of a lattice of irregular pentagons. Alternatively, the magnetic ions may be visualized as being enclosed by an edge-shared network of squares and equilateral triangles. (b) Sketch of the crystal and magnetic structure of $\mathrm{Bi}_{2} \mathrm{Fe}_{4} \mathrm{O}_{9}$ showing only $\mathrm{Fe}$ and $\mathrm{O}$ atoms and the $\mathrm{Fe}-\mathrm{O}-\mathrm{Fe}$ superexchange pathways. The $\mathrm{Fe}^{3}$ sites are the same as threefold symmetric sites in the Cairo lattice, while pairs of $\mathrm{Fe}^{4}$ spins occupy positions above and below the Cairo-lattice fourfold symmetric sites. (c) View of the crystal structure perpendicular to the $c$ axis, with $\mathrm{Bi}$ atoms in gray, $\mathrm{Fe}$ in yellow, and $\mathrm{O}$ in red.

fourfold sublattices are each individually ferromagnetic but are anti-aligned with each other. In between these limits, when $x \approx \sqrt{2}$, Rousochatzakis et al. [2] predict that a spin nematic phase would be realized for quantum spin.

In the two experimental realizations of the Cairo lattice studied in this work, $\mathrm{Bi}_{2} \mathrm{Fe}_{4} \mathrm{O}_{9}$ and $\mathrm{Bi}_{4} \mathrm{Fe}_{5} \mathrm{O}_{13} \mathrm{~F}$, the $\mathcal{J}_{33}$ interaction is expected to dominate due to the $180^{\circ} \mathrm{Fe}-\mathrm{O}-\mathrm{Fe}$ bond angle which is favorable for large antiferromagnetic superexchange interactions. In contrast, the other nearest neighbor interactions have $120^{\circ}-130^{\circ}$ bond angles which also leads to antiferromagnetic couplings but are much weaker. Indeed the orthogonal structure is observed at low temperatures in both $\mathrm{Bi}_{2} \mathrm{Fe}_{4} \mathrm{O}_{9}$ [3] and $\mathrm{Bi}_{4} \mathrm{Fe}_{5} \mathrm{O}_{13} \mathrm{~F}$ [4,5]. This orthogonal structure (expected in the case of low frustration index $x$ ) is noncollinear like the triangular $120^{\circ}$ structure, and may lead to interesting magnetic excitations, as was recently observed for the $120^{\circ}$ system (Y,Lu) $\mathrm{MnO}_{3}[6,7]$.

In order to verify the exchange interactions, and thus experimentally determine $x$, and to compare with the theoretical phase diagram of [2], we have measured the magnon spectrum of $\mathrm{Bi}_{2} \mathrm{Fe}_{4} \mathrm{O}_{9}$, and the related material $\mathrm{Bi}_{4} \mathrm{Fe}_{5} \mathrm{O}_{13} \mathrm{~F}$, which is formed by interleaving additional layers of $\mathrm{Fe}^{3+}(S=5 / 2)$ spins (denoted $\mathrm{Fe}^{\text {int }}$ ) in between the fourfold sites along $c$ between the pentagonal layers.

\section{METHODS}

Inelastic neutron scattering measurements were performed on single crystals of $\mathrm{Bi}_{2} \mathrm{Fe}_{4} \mathrm{O}_{9}$ on the triple-axis spectrometers EIGER (at the Paul-Scherrer-Institut, Switzerland) [8] and IN20 (at the Institut-Laue-Langevin, France) [9]. A co-aligned

array of six crystals was used for the EIGER measurements with total sample mass $\approx 0.6 \mathrm{~g}$, while the IN20 measurements used only the largest crystal with mass $0.28 \mathrm{~g}$. The crystals were grown in $\mathrm{Bi}_{2} \mathrm{O}_{3}$ flux in Pt crucibles, and their crystallinity and orientations were checked using the Bio-D diffractometer at HANARO, Korea [10]. The crystals were single phased and found to adopt the Pbam space group with lattice parameters $a=7.99 \AA, b=8.43 \AA$, and $c=6.01 \AA$, in agreement with the literature [3]. A small $15 \mathrm{mg}$ single crystal was used for high field magnetization measurements at ISSP, Japan, in pulsed fields up to $65 \mathrm{~T}$, with the crystals oriented with the field along [110] and [001] in turn.

In the EIGER measurements the crystals were mounted with $[h h 0]-[00 l]$ directions in the horizontal scattering plane, while on IN20 the [h00]-[0k0] directions were horizontal. In the IN20 measurements, small out-of-plane momentum transfers were accessible by tilting the cryostat. Both spectrometers were operated with fixed final energy $E_{F}=14.7 \mathrm{meV}$ in the $W$ configuration (scattering sense -1, $+1,-1$ at the monochromator, sample, and analyzer, respectively) with open collimation (without collimators between the axes). We used the double-focusing $\mathrm{Si}(111)$ monochromator and PG(002) analyzer on IN20, while a focused PG(002) monochromator and analyzer set up was used on EIGER. Both setups used a PG filter before the analyzer. Because of the lower flux of EIGER, measurements there were carried out at $200 \mathrm{~K}$, just below $T_{N}=245 \mathrm{~K}$, where the larger magnon population factor enhances the signal. In addition to unpolarized measurements [11] of the magnon dispersion at $2 \mathrm{~K}$ on IN20, we also measured the $X Y Z$ polarization dependence at selected Brillouin zone center and zone edge positions. In this case, the horizontally focused Heusler monochromator and analyzer was used with a flipper on the scattered beam and a PG filter in front of the analyzer. Helmholtz coils were used to align the neutron polarization to be parallel to the momentum transfer $(x)$, perpendicular to this in the horizontal scattering plane $(y)$ and vertically $(z)$.

Supplementary inelastic neutron scattering measurements [12] on polycrystalline powders of $\mathrm{Bi}_{2} \mathrm{Fe}_{4} \mathrm{O}_{9}$ were taken using the Merlin [13] and MAPS [14] time-of-flight (ToF) spectrometers at the ISIS facility, UK. The $20 \mathrm{~g}$ powder sample was synthesized from a solid state reaction in air and checked to be single phased using a Rigaku MiniFlex x-ray diffractometer. Merlin was operated in rep-rate multiplication mode with the gadolinium Fermi chopper running at $600 \mathrm{~Hz}$ and focused incident energies of 120 and $180 \mathrm{meV}$. On MAPS we used $E_{i}=300 \mathrm{meV}$ with the "sloppy" chopper running at $600 \mathrm{~Hz}$. Finally, inelastic neutron scattering measurements [15] on polycrystalline powders of $\mathrm{Bi}_{4} \mathrm{Fe}_{5} \mathrm{O}_{13} \mathrm{~F}$ were performed on the IN4 [16] ToF spectrometer at ILL, France. The $6.4 \mathrm{~g}$ powder sample was synthesized by a solid state reaction in evacuated sealed quartz tubes and used previously for diffraction measurements [4,5]. We used incident neutron energies of 150,66 , and $16.6 \mathrm{meV}$ with the Fermi chopper running at $333 \mathrm{~Hz}$ for 150 and $66 \mathrm{meV}$ and $200 \mathrm{~Hz}$ for $16.6 \mathrm{meV}$. Additional measurements at higher energies on the same sample was carried out using the MARI spectrometer at ISIS. The IN4 data was processed using the LAMP [17] software with subsequent checks using Mantid [18]. MAPS, Merlin, and MARI data were processed using Mantid. We 


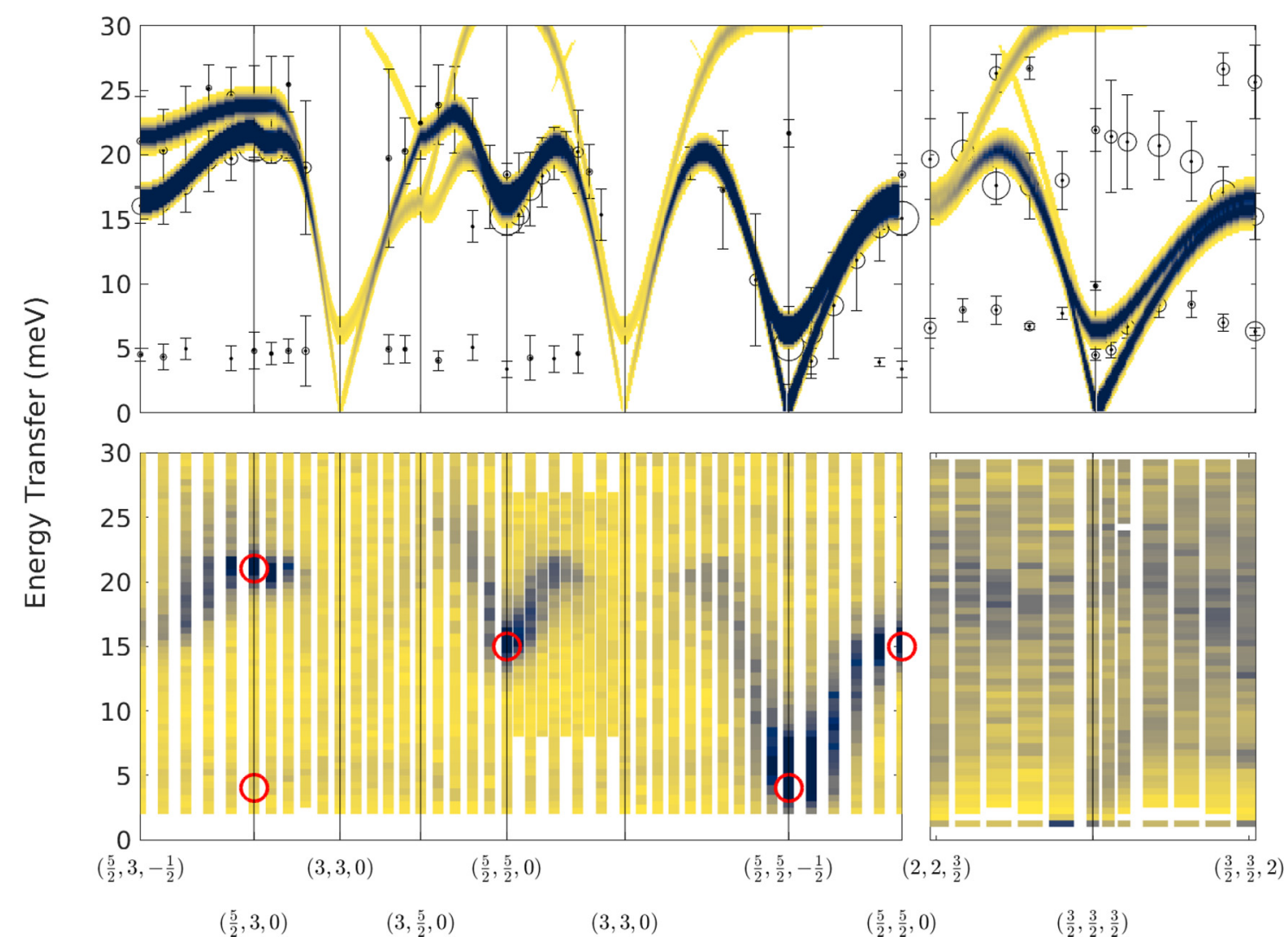

FIG. 2. The magnon dispersion of $\mathrm{Bi}_{2} \mathrm{Fe}_{4} \mathrm{O}_{9}$ at $2 \mathrm{~K}$ measured by inelastic neutron scattering on IN20 (bottom left), and calculated from the spin wave model described in the text (top left), together with that measured at $200 \mathrm{~K}$ on EIGER (right panels). Markers in the upper panels indicated the fitted peak positions with the marker size proportional to the fitted peak areas, and the error bars proportional to the fitted widths. The color map shows the measured $\chi^{\prime \prime}(\mathbf{Q}, \omega)$ on an arbitrary scale. The flat mode below $10 \mathrm{meV}$ and that around $20 \mathrm{meV}$ along $[00 L]$ in the Eiger data are believed to be phonons, while the other modes are well fitted by the spin wave model. Large red circles indicate the points where polarized neutron measurements were made.

used the SpinW $[19,20]$ and McPhase $[21,22]$ software packages to model the data.

\section{INELASTIC NEUTRON SCATTERING}

\section{A. $\mathrm{Bi}_{2} \mathrm{Fe}_{4} \mathrm{O}_{9}$}

Figure 2 shows the single-crystal unpolarized data for $\mathrm{Bi}_{2} \mathrm{Fe}_{4} \mathrm{O}_{9}$ collected on IN20 (at $2 \mathrm{~K}$ ) and EIGER (at $200 \mathrm{~K}$ ). In order to compare the two different temperatures, the measured scattering intensity $S(\mathbf{Q}, \omega)$ was converted to the dynamical susceptibility $\chi^{\prime \prime}(\mathbf{Q}, \omega)$ via the fluctuation-dissipation theo$\operatorname{rem} \chi^{\prime \prime}(\mathbf{Q}, \omega) \propto[1-\exp (-\beta \hbar \omega)] S(\mathbf{Q}, \omega)$ and it is $\chi^{\prime \prime}(\mathbf{Q}, \omega)$ which is plotted on an arbitrary scale. The measured dispersion at low temperature is very similar to a recent single crystal inelastic neutron scattering study [23]. Through most of the Brillouin zone, only a single intense dispersive mode was observed in the energy transfer range up to $30 \mathrm{meV}$. A much weaker, almost flat mode at around $5 \mathrm{meV}$ energy transfer was also seen on both IN20 and Eiger, and another faint dispersive mode between 15 and $25 \mathrm{meV}$ in the [00L] direction is seen in the Eiger data at $200 \mathrm{~K}$ but not in the IN20 data. These modes are not well described by linear spin wave theory (see Sec. IV), and are likely to be scattering from phonons. Another possibility which applies only to the higher energy modes is that they could arise from a spurious signal due to higher order scattering from the monochromator and analyzer. This " $2 k_{i}=3 k_{f}$ spurion" comes from the second order scattering of neutrons from the monochromator which matches the energy for third order scattering from the analyzer and appears as an apparent inelastic band at an energy transfer of $\frac{5}{4}$ of $E_{f}$, which in our case corresponds to $18.4 \mathrm{meV}$. This would only apply to the EIGER experiments where $\mathrm{PG}(002)$ was used for both the analyzer and monochromator. For the IN20 measurements, a Si(111) monochromator combined with a $\mathrm{PG}(002)$ analyzer was used, and since there is almost no half-wavelength contamination with $\mathrm{Si}(111)$, the $2 k_{i}$ spurion should be absent on IN20. In any case, such a spurious signal should be dispersionless and not change with $\mathbf{Q}$, whereas the signal we observed in the Eiger data does show some dispersion. Thus it is more likely to be a phonon than a spurious signal.

Table I summarizes the longitudinal polarization analysis measurements on IN20 at $2 \mathrm{~K}$ at selected reciprocal lattice positions $(\mathbf{Q}, \omega)$. The spectrometer was set to these positions and the cross sections with the neutron beam polarized along the directions $x \| \mathbf{Q}, y \perp \mathbf{Q}$ in the horizontal plane and $z$ vertical was measured in the spin-flip (SF) and non-spin-flip (NSF) channels. We did not perform a rocking scan but left the spectrometer at the fixed $(\mathbf{Q}, \omega)$ for the six measurements per position shown. From these cross sections, signals 
TABLE I. Longitudinal polarization analysis measurements at selected zone center and zone boundary positions. All values are counts per $4 \times 10^{6}$ monitor counts (approximately $10 \mathrm{~min}$ ), except for the measurement at $\left(\frac{5}{2} 30\right), 4 \mathrm{meV}$ which is in counts per $2 \times 10^{7}$ monitor counts. Numbers in parentheses are standard errors.

\begin{tabular}{|c|c|c|c|c|c|c|c|c|c|c|}
\hline$(h k l), \hbar \omega$ & $I_{x}^{\mathrm{NSF}}$ & $I_{x}^{\mathrm{SF}}$ & $I_{y}^{\mathrm{NSF}}$ & $I_{y}^{\mathrm{SF}}$ & $I_{z}^{\mathrm{NSF}}$ & $I_{z}^{\mathrm{SF}}$ & \multicolumn{2}{|c|}{$M_{y y}$} & \multicolumn{2}{|c|}{$M_{z z}$} \\
\hline$\left(\frac{5}{2} 30\right), 21 \mathrm{meV}$ & 41 & 388 & 46 & 347 & 335 & 71 & $5(9)$ & $41(27)$ & 294(19) & $317(21)$ \\
\hline$\left(\frac{5}{2} \frac{5}{2} 0\right), 15 \mathrm{meV}$ & 56 & 499 & 73 & 533 & 539 & 71 & $17(11)$ & $-34(32)$ & $483(24)$ & $428(24)$ \\
\hline
\end{tabular}

proportional to the spin fluctuations in the $y$ and $z$ directions may be determined using the equations [24]

$$
\begin{aligned}
M_{y y}=\left\langle M_{\perp y} M_{\perp y}^{\dagger}\right\rangle_{\omega} & =\sigma_{y}^{\mathrm{NSF}}-\sigma_{x}^{\mathrm{NSF}} \\
& =\sigma_{x}^{\mathrm{SF}}-\sigma_{y}^{\mathrm{SF}} \\
M_{z z}=\left\langle M_{\perp z} M_{\perp z}^{\dagger}\right\rangle_{\omega} & =\sigma_{z}^{\mathrm{NSF}}-\sigma_{x}^{\mathrm{NSF}} \\
& =\sigma_{x}^{\mathrm{SF}}-\sigma_{z}^{\mathrm{SF}},
\end{aligned}
$$

where $M_{\perp}$ is the component of the magnetic scattering vector perpendicular to $\mathbf{Q}$ and $\langle\cdots\rangle_{\omega}$ indicates the time Fouriertransformed thermal average. We have also assumed that the chiral $\left(\propto\langle\mathbf{M} \times \mathbf{M}\rangle_{\omega}\right)$ and nuclear-magnetic interference $(\propto$ $\langle N \mathbf{M}\rangle_{\omega}$, where $N$ is the nuclear scattering amplitude) terms in the cross section are negligible compared to the strong magnetic signal. This means that the incident polarization was always kept the same and only the flipper on the scattered beam was turned on (for SF) or off (for NSF). The measurements in Table I show that the low energy flat mode around $4-5 \mathrm{meV}$ is nonmagnetic, as indicated by the $\left(\frac{5}{2} 30\right), 4 \mathrm{meV}$ point, implying that this mode is most likely a phonon. The polarized measurements also show that the magnetic fluctuations in the zone center and zone boundaries are almost entirely out-of-plane (along $z \| c$ ), consistent with an easy plane single-ion anisotropy as will be discussed in Sec. IV.

In addition to the data below $30 \mathrm{meV}$ shown in Fig. 2, we had also measured peaks at around $35 \mathrm{meV}$ which appeared dispersive in the IN20 data. However, later analysis showed that this was an experimental artifact, arising from the inelastic incoherent scattering of a sample Bragg peak from the analyzer. Preliminary spin wave theory calculations based on exchange parameters previously calculated by density functional theory (DFT) [5,25] showed two bands of excitations, with the lower energy band associated with the interlayer exchange interaction and the upper coming from the in-plane interactions. As we had apparently seen both bands in scans on IN20 up to $40 \mathrm{meV}$, we did not extend the measurements to higher energies at the time. When subsequent analysis indicated that the upper mode is actually spurious scattering, we performed additional high energy measurements on powder samples.

Figures 3 and 4 show the data on $\mathrm{Bi}_{2} \mathrm{Fe}_{4} \mathrm{O}_{9}$ powder measured on MAPS and Merlin. Additional scattering which follows the magnetic form factor was observed at $\approx 40 \mathrm{meV}$ and between 60 and $80 \mathrm{meV}$. Modeling the data, we found that the relatively narrow flat mode at $40 \mathrm{meV}$ is associated with the in-phase precession of the ferromagnetically coupled

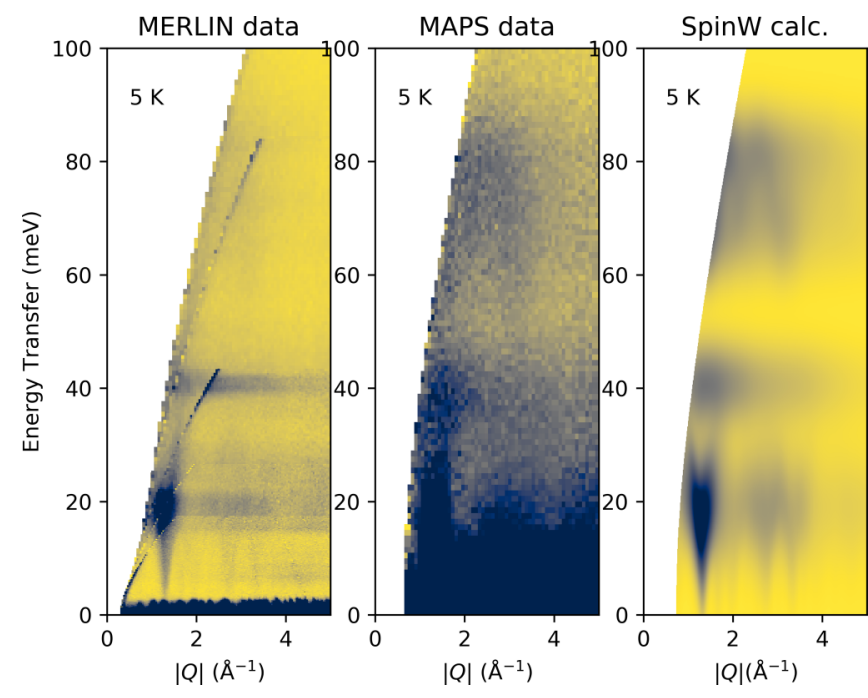

FIG. 3. Powder inelastic neutron scattering data on $\mathrm{Bi}_{2} \mathrm{Fe}_{4} \mathrm{O}_{9}$ from MERLIN and MAPS at $5 \mathrm{~K}$. Data from multiple MERLIN runs with $E_{i}=25,38,62,120$, and $180 \mathrm{meV}$ is shown overlapped on the left panel. MAPS data shows the $E_{i}=300 \mathrm{meV}$ run. Several magnon bands up to $90 \mathrm{meV}$ are visible as described in the text.

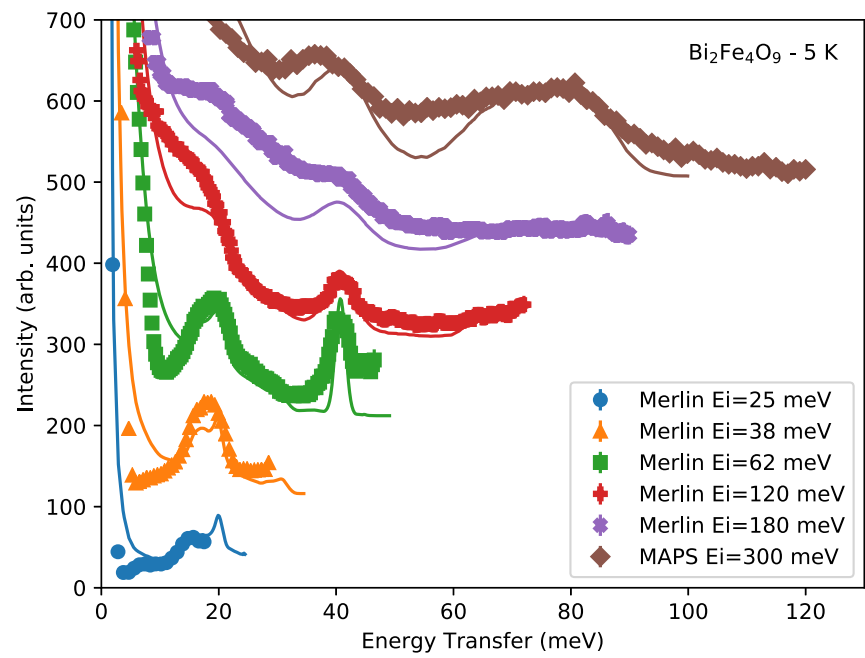

FIG. 4. Cuts along energy transfer of the powder inelastic neutron scattering data on $\mathrm{Bi}_{2} \mathrm{Fe}_{4} \mathrm{O}_{9}$ from MERLIN and MAPS at $5 \mathrm{~K}$, integrating over the low $|Q|$ region up to $3 \AA^{-1}$. Solid lines are fits to a spin wave model described in the text. 


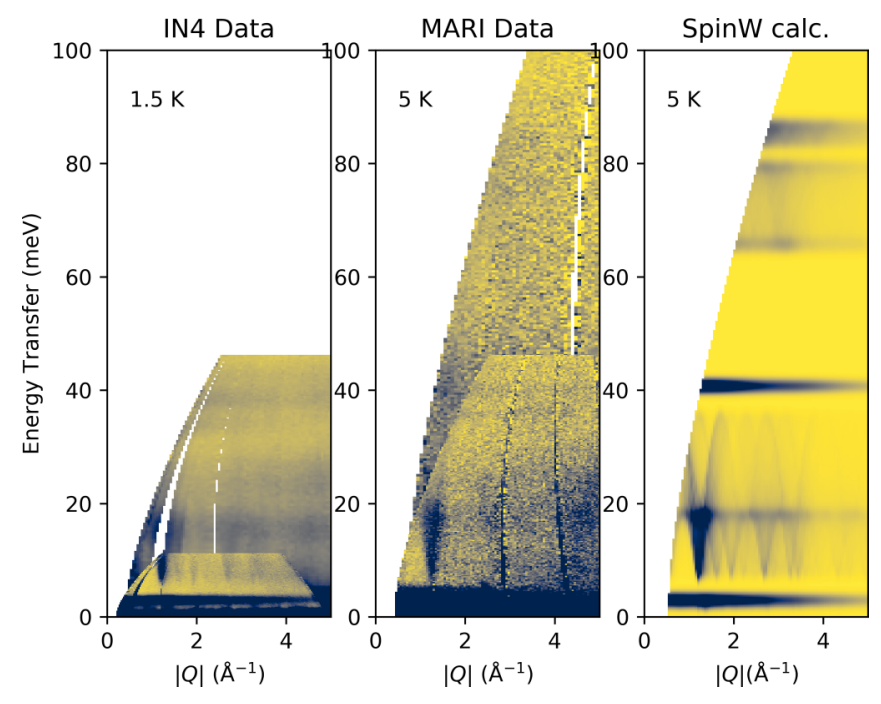

FIG. 5. Powder inelastic neutron scattering data on $\mathrm{Bi}_{4} \mathrm{Fe}_{5} \mathrm{O}_{13} \mathrm{~F}$ from IN4 and MARI at low temperatures. Data from multiple runs with $E_{i}=16.6$ and $66 \mathrm{meV}$ from IN4 and $E_{i}=66$ and $160 \mathrm{meV}$ from MARI is shown overlapped. Several magnon bands up to $90 \mathrm{meV}$ similar to $\mathrm{Bi}_{2} \mathrm{Fe}_{4} \mathrm{O}_{9}$ are observed as described in the text.

spins which form the fourfold $\mathrm{Fe}^{4}$ site in the Cairo lattice. The energy of this mode thus serves to pin down the $J_{44}$ exchange parameter which couples these spins. The in-plane dimer interaction $J_{33}$ on the other hand determines the energy separation between the lower and upper magnon bands. That we observed the upper band at relatively high energies (between 60 and $80 \mathrm{meV}$ ) thus implies a large $\left|J_{33}\right|$ which is significantly larger than anticipated by DFT calculations. Finally, the bandwidths of the magnon bands are determined by the other in-plane interactions (both upper and lower bands) and the interlayer coupling $J_{c}$ (lower band only).

\section{B. $\mathrm{Bi}_{4} \mathrm{Fe}_{5} \mathrm{O}_{13} \mathrm{~F}$}

Figures 5 and 6 show the inelastic neutron spectrum of powder $\mathrm{Bi}_{4} \mathrm{Fe}_{5} \mathrm{O}_{13} \mathrm{~F}$ as $2 \mathrm{D}$ contour maps and energy cuts, respectively. Magnetic excitations are seen up to $\approx 80 \mathrm{meV}$ like in $\mathrm{Bi}_{2} \mathrm{Fe}_{4} \mathrm{O}_{9}$. Similarly, the pattern of a strongly dispersive band below $20 \mathrm{meV}$, a flat band around $40 \mathrm{meV}$, and another band at higher energies is also seen. The largest difference between the spectra of the two materials is an intense flat mode below the dispersive mode at $\approx 3 \mathrm{meV}$. This low energy mode is observed only at low temperatures in phase I, and disappears above the $T_{1}=62 \mathrm{~K}$ transition, as shown in Fig. 7. It is thus associated with ordering of the intermediate $\mathrm{Fe}^{\text {int }}$ layer, which is supported by spin wave modeling where the mode disappears if the moments on the $\mathrm{Fe}^{\mathrm{int}}$ site is disordered.

In addition, the $40 \mathrm{meV}$ flat band in $\mathrm{Bi}_{2} \mathrm{Fe}_{4} \mathrm{O}_{9}$ appears to be split into two bands in $\mathrm{Bi}_{4} \mathrm{Fe}_{5} \mathrm{O}_{13} \mathrm{~F}$. Like in $\mathrm{Bi}_{2} \mathrm{Fe}_{4} \mathrm{O}_{9}$, this mode is associated with the ferromagnetically coupled fourfold symmetric sites $\mathrm{Fe}^{4}$. In addition, these ions are coupled to the intermediate layer $\mathrm{Fe}^{\text {int }}$ sites by the interlayer $J_{c}$ interaction. However, this antiferromagnetic interaction is not enough to lead to a splitting of the $40 \mathrm{meV}$ band. As explained in the next section, this splitting could be described in spin wave theory only if the intermediate layer $\mathrm{Fe}^{\mathrm{int}}$ spins are

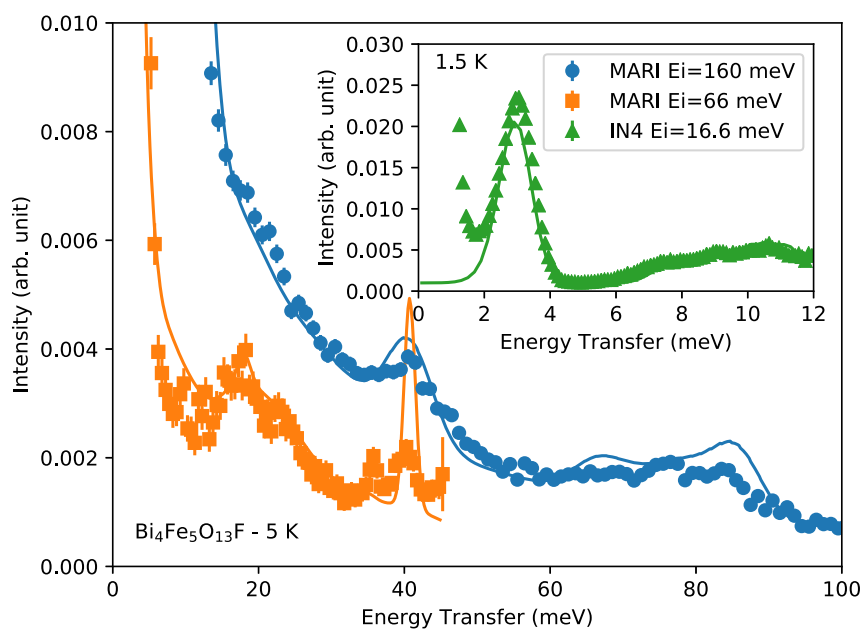

FIG. 6. Cuts along energy transfer of the powder inelastic neutron scattering data on $\mathrm{Bi}_{4} \mathrm{Fe}_{5} \mathrm{O}_{13} \mathrm{~F}$ from IN4 and MARI at low temperatures, integrating over a narrow region around the first magnetic Brillouin zone center. Solid lines are spin wave model fits.

canted with respect to the $\mathrm{Fe}^{4}$ spins. Since neutron diffraction measurements show that this is not the case, we have been unable to model this splitting in the data.

\section{SPIN WAVE THEORY}

The exchange interaction topology for the two materials is shown in Fig. 8. We found that a Heisenberg spin Hamiltonian with isotropic exchange interaction and single-ion anisotropy is enough to model the measured excitations,

$$
\mathcal{H}=\sum_{i j} J_{i j} \mathbf{S}_{i} \cdot \mathbf{S}_{j}+\sum_{i} A_{i} S_{z}^{2}(i)
$$

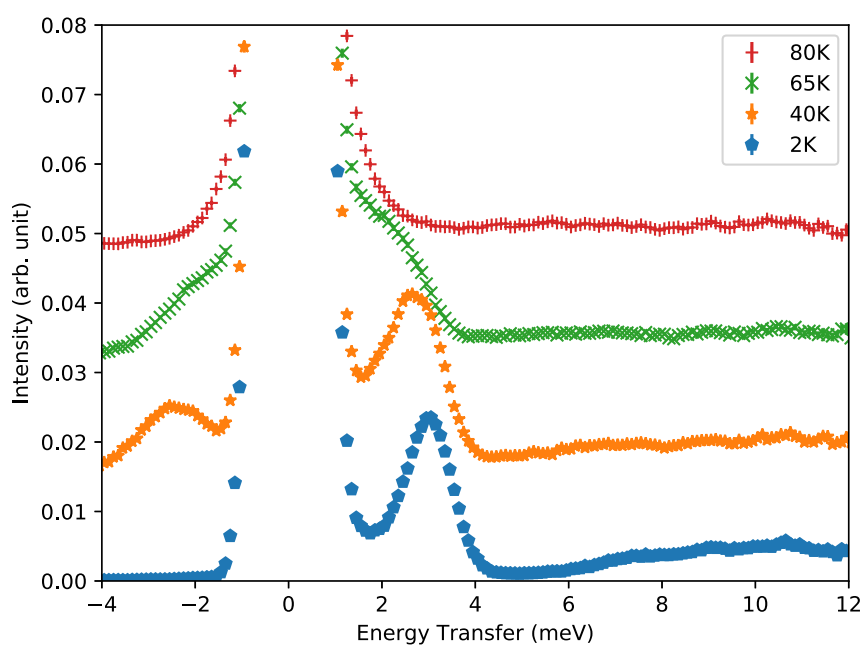

FIG. 7. Temperature dependence of the scattering around the first Brillouin zone center as a function of temperature, as measured on IN4 with $E_{i}=16.6 \mathrm{meV}$. The mode at $\approx 3 \mathrm{meV}$ is associated with the intermediate layer spins which become disordered above $T_{1}=62 \mathrm{~K}$ : it is clearly present in the low temperature phase below $T_{1}$, becomes quasielastic in the intermediate phase between $T_{1}$ and $T_{2}=71 \mathrm{~K}$, and disappears above $T_{2}$. 


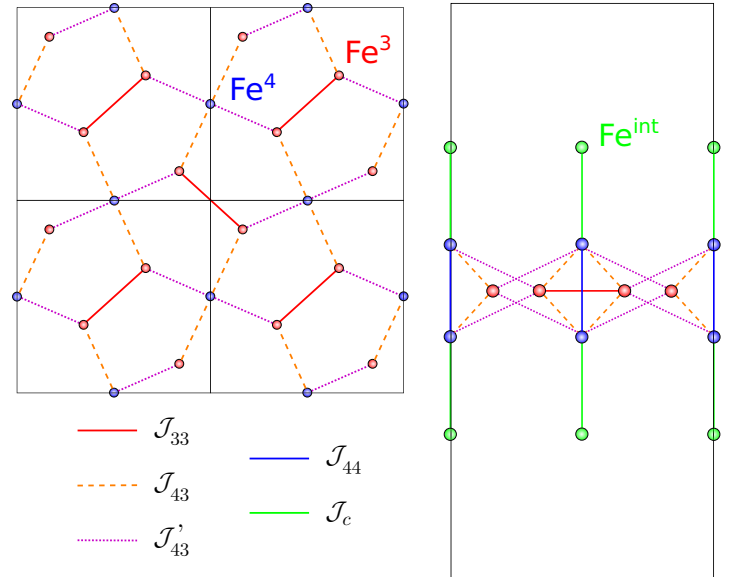

FIG. 8. Exchange couplings between $\mathrm{Fe}^{3+}$ spins in the Cairo lattice compounds. Blue circles indicate the fourfold sites $\mathrm{Fe}^{4}$, red circles are the threefold sites $\mathrm{Fe}^{3}$, and green circles are the intermediate layer $\mathrm{Fe}^{\mathrm{int}}$ sites in $\mathrm{Bi}_{4} \mathrm{Fe}_{5} \mathrm{O}_{13} \mathrm{~F}$. In $\mathrm{Bi}_{2} \mathrm{Fe}_{4} \mathrm{O}_{9}$ there are no $\mathrm{Fe}^{\mathrm{int}}$ sites, and instead the $J_{c}$ couplings link $\mathrm{Fe}^{4}$ sites on adjacent pentagon layers.

where positive $J>0$ indicates antiferromagnetic interactions and positive $A>0$ indicates an easy-plane single-ion anisotropy (SIA).

Following $a b$ initio calculations [5] which indicated that there are preferred easy axes for each spin site within the $a-b$ plane, we have also modeled the case where $A<0$ is negative where the spins prefer to lie parallel or antiparallel to an easyaxis direction along the bond between threefold and fourfold sites. However, the modeling shows that both axial and planar anisotropy scenarios can give almost the same dispersion and intensities (including polarized cross sections at the measured zone center and zone boundary points) albeit with different values of $|A|$.

The exception to this is at low energies close to the zone center or zone boundary, where in the easy-plane case the acoustic magnon is split into two modes. One mode (involving precession out of the plane) is gapped, while the other modes (where the spins only precess within the plane) remains gapless. In the easy-axis case, there is only a single gapped acoustic mode since any precession away from the preferred direction costs energy. However, due to the presence of the magnetic Bragg peak and the incoherent elastic line it is hard to discern the presence of one or two modes. The measured linewidths of the constant- $Q$ scans is quite broad (FWHM $\approx 6 \mathrm{meV}$, compared to an instrumental resolution of $\approx 1.5 \mathrm{meV}$ ) and could be fitted by two peaks but there is no distinct minimum between them. Polarized measurements at the zone boundary acoustic modes [e.g., (330) $4 \mathrm{meV}$ ] would have been conclusive but the weak signal and lack of time meant we could not do this. If the anisotropy is planar, this measurement would show that the magnetic fluctuations are polarized in the $a-b$ plane, while if the anisotropy is axial, the polarized measurement would show fluctuations to be mainly out-of-plane.

Since our data cannot distinguish between the easy-plane and easy-axis models, we have chosen to use a spin wave model with a single easy-plane anisotropy term for $\mathrm{Bi}_{2} \mathrm{Fe}_{4} \mathrm{O}_{9}$ for all sites for simplicity in the following analysis. In the case of $\mathrm{Bi}_{4} \mathrm{Fe}_{5} \mathrm{O}_{13} \mathrm{~F}$, the DFT calculations show that the magnitude of the SIA on the intermediate $\mathrm{Fe}^{\text {int }}$ sites is much larger than on the others. We have thus modeled this with two easy-plane SIA terms, one for sites in the Cairo-lattice plane and one for the intermediate layer sites.

In terms of the exchange interactions, the real systems $\mathrm{Bi}_{2} \mathrm{Fe}_{4} \mathrm{O}_{9}$ and $\mathrm{Bi}_{4} \mathrm{Fe}_{5} \mathrm{O}_{13} \mathrm{~F}$ deviate from the ideal Cairo pentagonal lattice in several respects. First, the fourfold site is occupied not by a single $\mathrm{Fe}^{3+}$ spin, but rather by a pair of spins (each denoted $\mathrm{Fe}^{4}$ in Fig. 1(b) and Fig. 8) offset above and below the pentagonal plane. This leads additionally to an exchange interaction $J_{44}$ between these spins. Second, due to an asymmetry in the positions of the oxygen ligands, there are two different $\mathcal{J}_{43}$ interactions connecting the fourfold sites and the threefold sites, denoted $J_{43}$ and $J_{43}^{\prime}$. Finally, there is a finite interlayer interaction $J_{c}$ through the fourfold sites. In $\mathrm{Bi}_{4} \mathrm{Fe}_{5} \mathrm{O}_{13} \mathrm{~F}$ this interaction connects the fourfold sites above and below the pentagonal plane with an intermediate layer of $\mathrm{Fe}^{3+}$ spins, denoted $\mathrm{Fe}^{\text {int }}$ in Fig. 8. The threefold sites are denoted $\mathrm{Fe}^{3}$. We use this notation to ensure a uniform description of the different sites in the physical compounds and the theoretical Cairo lattice, and to reconcile the different notations in the literature for $\mathrm{Bi}_{2} \mathrm{Fe}_{4} \mathrm{O}_{9}$ and $\mathrm{Bi}_{4} \mathrm{Fe}_{5} \mathrm{O}_{13} \mathrm{~F}$ [26].

As noted in the previous section, the in-plane Cairo dimer interaction $J_{33}$ splits the dispersive modes into two bands. The upper band involves mostly precession of the spins on the threefold sites. These spins are antiferromagnetically aligned, so that as $J_{33}$ becomes larger in the positive sense (becomes more antiferromagnetic), this configuration is increasingly stabilized so that it costs more energy to cause the spins to precess away from rigid anti-alignment. Our observation that the upper bands are between 60 and $80 \mathrm{meV}$ thus points to a surprisingly large antiferromagnetic $J_{33}$, in agreement with [23].

The out-of-phase precession of the ferromagnetically aligned pair of spins above and below the Cairo fourfold site yields a flat band at intermediate energy. A larger ferromagnetic (more negative) $J_{44}$ exchange between these spins will cost this out-of-phase motion more energy and hence push the mode up in energy, while more antiferromagnetic $J_{44}$ will push it down in energy. We observed a flat mode at around $40 \mathrm{meV}$ which points to a moderate ferromagnetic $J_{44}$ in contrast with DFT calculations. It is also counter to the findings of Beauvois et al. [23] because they posit the flat band to be at $\approx 19 \mathrm{meV}$ and so deduced a moderate antiferromagnetic $J_{44}$. However, $19 \mathrm{meV}$ is very close to an energy transfer of $18.4 \mathrm{meV}$, which corresponds to a well-known (so-called " $2 k_{i}=3 k_{f}$ ") spurion of a triple-axis spectrometer operating with a final wave vector of $k_{f}=2.662 \AA^{-1}$, as was the case in [23]. We note that we also observe scattering at around this energy in our EIGER data which does not fit the spin wave model and may also be due to this same spurion.

In $\mathrm{Bi}_{4} \mathrm{Fe}_{5} \mathrm{O}_{13} \mathrm{~F}$ at low temperatures we apparently observe at least two flat bands around $40 \mathrm{meV}$, as shown in Figs. 5 and 6 . If these bands are magnetic, they may only be explained in terms of spin wave theory by a canting of the intermediate site $\mathrm{Fe}^{\mathrm{int}}$ spins with respects to the fourfold site $\mathrm{Fe}^{4}$ spins. This would only arise if there is a very strong easy-axis singleion anisotropy which would dominate over the exchange 
TABLE II. Spin wave exchange parameters fitted to data in meV. Positive values indicate antiferromagnetic exchange. $A$ denotes the single-ion anisotropy term on the threefold and fourfold sites of the Cairo plane, while $A^{\prime}$ is the term which applies to the intermediate layer spins in $\mathrm{Bi}_{4} \mathrm{Fe}_{5} \mathrm{O}_{13} \mathrm{~F}$ only.

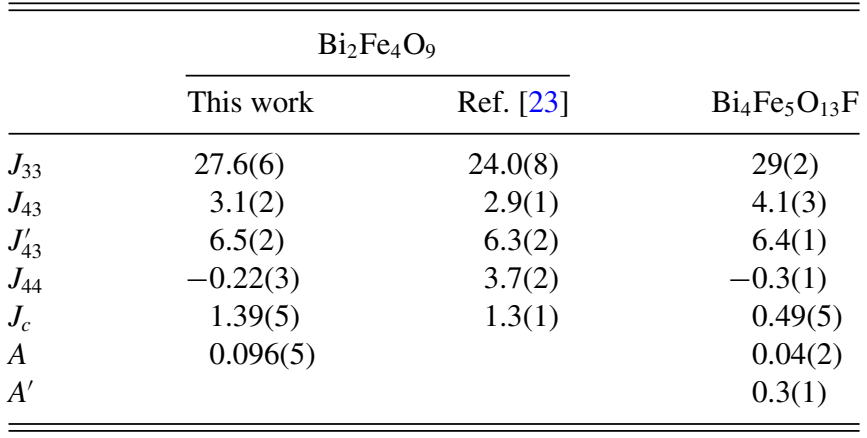

interaction $J_{c}$ between these spins which tends to keep them in (anti-)alignment. We calculated that $A$ should be an order of magnitude larger than $J_{c}$ for this to pertain, which seems physically unlikely, given that the $\mathrm{Fe}^{3+}$ ion has an $L=0$ ground state. Moreover, neutron diffraction [5] measurements find that these spins (on sites $\mathrm{Fe}^{4}$ and $\mathrm{Fe}^{\mathrm{int}}$ ) are antiparallel in phase I at low temperature, with no canting. There are also several optic phonon modes at these energies, but it is hard to discern if any of these modes are magnetic or phononic, because all modes show an initial decrease in intensity from 1 to $4 \AA^{-1}$ followed by an increase in intensity above $6 \AA^{-1}$. Because of the unlikelihood of a large easy-axis single-ion anisotropy and since it would yield a magnetic structure contrary to that observed experimentally, we have chosen the model with modest SIA which yields only a single flat mode.

Also in $\mathrm{Bi}_{4} \mathrm{Fe}_{5} \mathrm{O}_{13} \mathrm{~F}$ there is a low energy flat mode at $\approx 3 \mathrm{meV}$ which is due to precession of the intermediate layer $\mathrm{Fe}^{\text {int }}$ spins. The energy of this mode depends on $J_{c}$ and the SIA on the $\mathrm{Fe}^{\text {int }}$ spins $A^{\prime}$, while $J_{c}$ also affects the lower magnon band between $6-25 \mathrm{meV}$. We found that to fit both these features required a relatively large $A^{\prime}=0.3 \mathrm{meV}$ compared to the value in $\mathrm{Bi}_{2} \mathrm{Fe}_{4} \mathrm{O}_{9}$ of $A^{\prime}=0.096 \mathrm{meV}$, which is itself surprising for $\mathrm{Fe}^{3+}$ which has a $L=0$ ground state and is needed to explain the $\approx 5 \mathrm{meV}$ anisotropy gap in $\mathrm{Bi}_{2} \mathrm{Fe}_{4} \mathrm{O}_{9}$. However, the large value of $A^{\prime}$ compared to $A$ is consistent with DFT calculations of the SIA in $\mathrm{Bi}_{4} \mathrm{Fe}_{5} \mathrm{O}_{13} \mathrm{~F}$ [5] which found that the magnitude of SIA on the $\mathrm{Fe}^{\mathrm{int}}$ site is over 5 times that on the other sites.

Finally, the bandwidths of the lower and upper bands are determined by $J_{43}$ and $J_{43}^{\prime}$, while the lower bands are additionally modulated by $J_{c}$, since the upper bands involve mostly the threefold sites which are not coupled by $J_{c}$.

Taking these considerations into account together with the observed energies of the different magnon bands in the two compounds, we were able to obtain suitable starting parameters for a local minimum simplex search. The fitted exchange interactions are given in Table II. Neutron data files, spin wave models, and data analysis scripts may be obtained from [27].

\section{HIGH FIELD MAGNETIZATION}

Figure 9 shows the high field magnetization measured in a pulsed field and corresponding mean-field calculations.

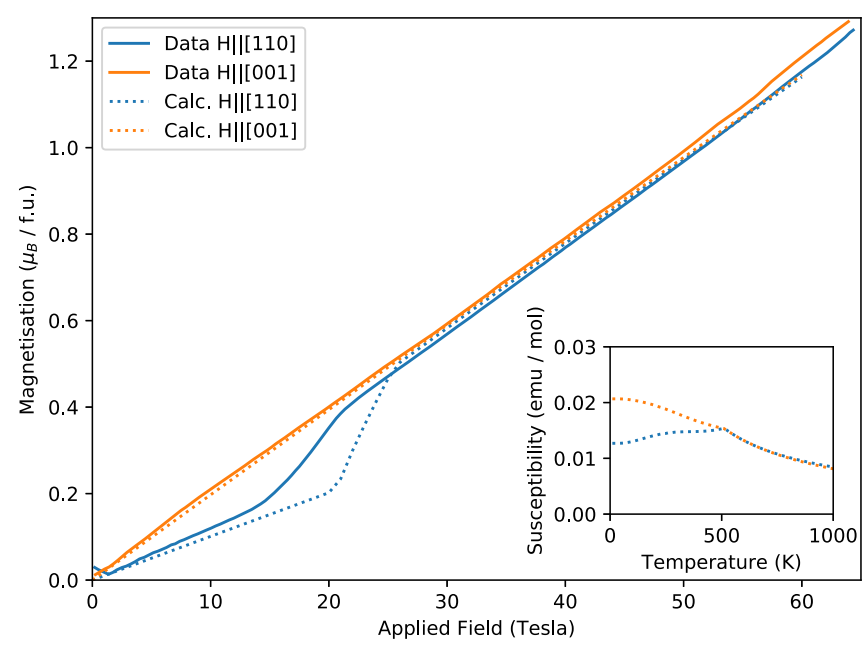

FIG. 9. High-field magnetization of $\mathrm{Bi}_{2} \mathrm{Fe}_{4} \mathrm{O}_{9}$ measured in a pulse field parallel to the [110] and [001] directions (solid lines) and corresponding mean-field calculations (dotted lines). The calculated curves have been scaled to the measured ordered moment of $\mathrm{Bi}_{2} \mathrm{Fe}_{4} \mathrm{O}_{9}$. The inset shows the calculated susceptibility at a field of $0.1 \mathrm{~T}$. A spin-flop transition is observed at $\approx 18 \mathrm{~T}$ in the data. In the model this critical field depends on the single-ion anisotropy. The SIA determined from a point charge model (see text) yields a critical field slightly higher than the observation but is qualitatively similar. The slight upturn in the data curve for $H \|[110]$ at low fields is a measurement artifact.

A metamagnetic transition is observed around $18 \mathrm{~T}$, and the mean-field calculations reveal it to be a spin-flop transition where the spins cant out of the $a-b$ plane in order to minimize the Zeeman energy of the in-plane field. The critical field depends primarily on the single-ion anisotropy which keeps the spins within the $a-b$ plane, and to a lesser extent on the exchange interactions which prefers the orthogonal Cairo magnetic structure.

The mean-field calculations used the exchange parameters fitted from the INS data as described above, but instead of a simple easy plane anisotropy term, a point charge model was used to calculate the full crystalline electric field. This is needed to use the intermediate coupling scheme which includes the effect of the on-site Coulomb and spin-orbit interactions, which we deemed to be more accurate than a simple spin-only approach. We used the nominal charges $(+3|e|$ for $\mathrm{Bi}$ and $\mathrm{Fe}$ and $-2|e|$ for $\mathrm{O}$ ) and calculated the effect of these charges on the magnetic $3 d$ electrons of the Fe sites by including contributions from neighboring charges up to $6.5 \AA$ from a given $\mathrm{Fe}$ site, which covers up to second neighboring $\mathrm{Fe}$ ions. These calculations found that the crystal field splitting on the threefold and fourfold sites had similar magnitude, yielding an overall splitting of $\approx 0.4 \mathrm{meV}$. This would correspond to a planar SIA term of $A_{\mathrm{pc}}=0.06 \mathrm{meV}$, which is $60 \%$ of the value found from fitting the spin anisotropy gap. Scaling up the magnitude of the crystal field splitting to match the measured spin anisotropy gap would increase the spin-flop transition field by an equivalent amount. Nonetheless, the chosen point charge model still overestimates the critical field of the spinflop transition, by $\approx 20 \%$. 
In any case, the point charge calculations showed that additional anisotropy is present in the $a b$ plane, with the threefold sites preferring to align at $52^{\circ}$ to the $a$ axis, and at $90^{\circ}, 180^{\circ}$, and $270^{\circ}$ to this angle. The fourfold sites, on the other hand, preferred to align parallel and antiparallel to $c$ but are kept pinned to the $a-b$ plane by the exchange coupling with the threefold sites. This is in contrast to the DFT calculations which found that all the moments preferred to lie within the $a-b$ plane. We should note here that the point charge model is quite a coarse model and does not include many effects such as charge transfer and covalency which the DFT calculation does take into account.

In addition, in order to match the measured slope of the magnetization, we found that we needed to scale the meanfield calculations (which assumed a full ordered moment of $5 \mu_{B} / \mathrm{Fe}$ ) by the ordered moment obtained by neutron diffraction measurements [3], $\approx 3.5 \mu_{B}$. The scaled magnetization is shown in Fig. 9. We also calculated the temperature dependence of the magnetization in a $0.1 \mathrm{~T}$ field, shown as an inset to Fig. 9. Because of the large $J_{33}$ interaction between pairs of threefold symmetric spins, we have used a cluster mean-field method to calculate $M(T)$. In the calculation, the pairs joined by $J_{33}$ are treated as a cluster using exact diagonalization, but other interactions are treated in the mean-field approximation. This reduces the calculated $T_{N}$ compared with a pure mean-field calculation but still retains some effects of $J_{33}$. The results agrees qualitatively with the measurements of Ressouche et al. [3] but overestimates the ordering temperature by a factor of 2 , yielding $T_{N}^{\mathrm{cmf}}=510 \mathrm{~K}$. In contrast, a pure mean-field calculation gives $T_{N}^{\mathrm{cmf}}=1150 \mathrm{~K}$.

Finally, we also applied the point charge calculations to $\mathrm{Bi}_{4} \mathrm{Fe}_{5} \mathrm{O}_{13} \mathrm{~F}$, and found again that the fourfold sites preferred to orient along $c$, while the threefold sites preferred an inplane direction offset $22^{\circ}$ to the $a$ axis, and the intermediate layers to align along the $\langle 110\rangle$ directions.

\section{DISCUSSIONS}

In agreement with previous INS measurements [23], we find a very strong dimer interaction $J_{33}$ between the threefold symmetric sites of the Cairo lattice in both $\mathrm{Bi}_{2} \mathrm{Fe}_{4} \mathrm{O}_{9}$ and $\mathrm{Bi}_{4} \mathrm{Fe}_{5} \mathrm{O}_{13} \mathrm{~F}$, which puts these materials firmly within the orthogonal phase of the theoretical phase diagram. In both compounds the values of $J_{33}$ deduced from the spin wave spectra is almost three times that calculated from DFT $[4,5]$, while the deduced value of the other exchange interactions are of comparable magnitudes to the theoretical value. The large $J_{33}$ also pushes the calculated mean-field ordering temperature to above $1000 \mathrm{~K}$, but goes some way to explaining the large Curie-Weiss temperature $\theta_{\mathrm{CW}} \approx-1670 \mathrm{~K}$ (the sum of the deduced exchanges for $\mathrm{Bi}_{2} \mathrm{Fe}_{4} \mathrm{O}_{9}$ is $-1585 \mathrm{~K}$ ). We thus also used a cluster mean-field method that treats the dimer linked by $J_{33}$ as a cluster and applies exact diagonalization to this cluster, which is then coupled to the other spins in a mean-field fashion. This calculation reduces the calculated $T_{N}^{\mathrm{cmf}}=510$ $\mathrm{K}$ which is still over twice the measured $T_{N}=245 \mathrm{~K}$. Given the large $J_{33}$, one would expect the dimers to still be strongly coupled well above the overall ordering temperature $T_{N}$. As noted by Beauvois et al. [23], this would lead to a correlated paramagnetic state which could give rise to novel phenomena.
The deduced exchange parameters for $\mathrm{Bi}_{2} \mathrm{Fe}_{4} \mathrm{O}_{9}$ and $\mathrm{Bi}_{4} \mathrm{Fe}_{5} \mathrm{O}_{13} \mathrm{~F}$ are quite similar, with the main difference being the deduced interlayer coupling, which was found to be $J_{c}=$ $1.39 \mathrm{meV}$ in $\mathrm{Bi}_{2} \mathrm{Fe}_{4} \mathrm{O}_{9}$ and $J_{c}=0.49 \mathrm{meV}$ in $\mathrm{Bi}_{4} \mathrm{Fe}_{5} \mathrm{O}_{13} \mathrm{~F}$. This could perhaps explain part of the decrease in ordering temperature from $T_{N}=238 \mathrm{~K}$ in $\mathrm{Bi}_{2} \mathrm{Fe}_{4} \mathrm{O}_{9}$ to $T_{N}=178 \mathrm{~K}$ in $\mathrm{Bi}_{4} \mathrm{Fe}_{5} \mathrm{O}_{13} \mathrm{~F}$, as the in-plane interactions in $\mathrm{Bi}_{4} \mathrm{Fe}_{5} \mathrm{O}_{13} \mathrm{~F}$ are deduced to be slightly larger than in $\mathrm{Bi}_{2} \mathrm{Fe}_{4} \mathrm{O}_{9} . \mathrm{Bi}_{4} \mathrm{Fe}_{5} \mathrm{O}_{13} \mathrm{~F}$ should thus also show the same correlated paramagnetic behavior as $\mathrm{Bi}_{2} \mathrm{Fe}_{4} \mathrm{O}_{9}$, but at a slightly lower temperature.

Finally, in contrast to the expectation of the $L=0$ ground state of the $\mathrm{Fe}^{3+}$ ion, we found that there is a non-negligible single-ion anisotropy in both $\mathrm{Bi}_{2} \mathrm{Fe}_{4} \mathrm{O}_{9}$ and $\mathrm{Bi}_{4} \mathrm{Fe}_{5} \mathrm{O}_{13} \mathrm{~F}$. This manifests in $\mathrm{Bi}_{2} \mathrm{Fe}_{4} \mathrm{O}_{9}$ in a spin anisotropy gap of $\approx 5 \mathrm{meV}$ and a spin-flop transition at a critical field of $\approx 17 \mathrm{~T}$. The gap is not directly measurable in $\mathrm{Bi}_{4} \mathrm{Fe}_{5} \mathrm{O}_{13} \mathrm{~F}$ because of the low energy flat mode at $\approx 3 \mathrm{meV}$ due to precession of intermediate layer spins in the lowest temperature ordered phase. However, fitting the energy of this mode yields an even higher SIA than for $\mathrm{Bi}_{2} \mathrm{Fe}_{4} \mathrm{O}_{9}$, which reflects the more distorted octahedral environment of the $\mathrm{Fe}^{\mathrm{int}}$ spins, and is in agreement with the larger SIA found for these sites by DFT calculations [5]. This gives support to the model of Tsirlin et al. [5] for the SIA being the origin of the series of phase transitions in $\mathrm{Bi}_{4} \mathrm{Fe}_{5} \mathrm{O}_{13} \mathrm{~F}$.

In this model the weak interlayer couplings means that the intermediate layer $\mathrm{Fe}^{\text {int }}$ spins are more susceptible to thermal fluctuations and become disordered first, at $T_{1}=62 \mathrm{~K}$. Below this temperature, the large SIA on these $\mathrm{Fe}^{\text {int }}$ spins combined with the $J_{c}$ coupling constrains the $\mathrm{Fe}^{4}$ spins to be antiparallel to the $\mathrm{Fe}^{\text {int }}$ spins. The intralayer interactions then favors a orthogonal magnetic structure with a particular mutual arrangement of adjacent $\mathrm{Fe}^{4}$ spins described by a local vector chirality introduced in Ref. [5]. However, the intrinsic SIA of the $\mathrm{Fe}^{3}$ and $\mathrm{Fe}^{4}$ sites prefers a different arrangement of the $\mathrm{Fe}^{4}$ spins described by the opposite sign of the local vector chirality, which is stabilized at high temperatures when the $\mathrm{Fe}^{\mathrm{int}}$ spins become disordered, in the phase between $T_{2}=71$ $\mathrm{K}$ and $T_{N}=178 \mathrm{~K}$. In between is a phase where the direction of preferred orientations of the $\mathrm{Fe}^{4}$ spins determined by the competing SIA and intralayer interactions crosses over giving a collinear, partial-ordered structure in the narrow temperature window between $T_{1}=62 \mathrm{~K}$ and $T_{2}=71 \mathrm{~K}$. Although this collinear structure is very similar to the theoretically predicted structure in the limit of small $\mathcal{J}_{43} / \mathcal{J}_{33}$ [2], it does not have the same origin. In the theory of Rousochatzakis et al. [2], which is based on a Heisenberg Hamiltonian without single-ion anisotropy, the collinear phase is driven by quantum fluctuations. Whereas in the model of Tsirlin et al. [5] it is a crossover between two orthogonal ordered phases with opposite local vector chiralities, necessitating a collinear state in between. Because we have a high $\operatorname{spin}(S=5 / 2)$ system, the threshold for the quantum fluctuations driven model is $\mathcal{J}_{43} / \mathcal{J}_{33} \lesssim 0.1$ [5]. Our larger value for $J_{33}$ than the DFT calculated values used by Tsirlin et al. [5] suggests that we are approaching this limit in this case $\left(J_{43} / J_{33}=0.11\right.$ and $\left.J_{43}^{\prime} / J_{33}=0.24\right)$. However, the experimental evidence for a relatively large single-ion anisotropy and the fact that the collinear phase exists only in a narrow range of temperature between two orthogonal phases indicates that the single-ion 
anisotropy driven model for the origin of this phase is correct for $\mathrm{Bi}_{4} \mathrm{Fe}_{5} \mathrm{O}_{13} \mathrm{~F}$. This also suggests that there is scope for further theoretical work to extend the phase diagram to include a single-ion anisotropy term, in order to better account for the behavior of the materials which realize the Cairo lattice.

\section{ACKNOWLEDGMENTS}

The work at the IBS CCES was supported by the research program of Institute for Basic Science (IBS-R009-G1). A.T. was supported by the Federal Ministry for Education and
Research through the Sofja Kovalevskaya Award of Alexander von Humboldt Foundation. Work of J.G.P. was supported by the Leading Researcher Program of the National Research Foundation of Korea (Grant No. 2020R1A3B2079375). Inelastic neutron scattering experiments were performed at the reactor of the Institute Laue-Langevin, Grenoble, France, at the Swiss spallation neutron source SINQ, at the Paul Scherrer Institute, Villigen, Switzerland, and at the ISIS Neutron and Muon Source, UK. Experiments at ISIS were supported by a beamtime allocation STFC RB1820598 from the Science and Technology Facilities Council.
[1] C. Lacroix, P. Mendels, and F. Mila, Introduction to Frustrated Magnetism: Materials, Experiments, Theory (Springer, Berlin, 2011).

[2] I. Rousochatzakis, A. M. Läuchli, and R. Moessner, Phys. Rev. B 85, 104415 (2012).

[3] E. Ressouche, V. Simonet, B. Canals, M. Gospodinov, and V. Skumryev, Phys. Rev. Lett. 103, 267204 (2009).

[4] A. M. Abakumov, D. Batuk, A. A. Tsirlin, C. Prescher, L. Dubrovinsky, D. V. Sheptyakov, W. Schnelle, J. Hadermann, and G. Van Tendeloo, Phys. Rev. B 87, 024423 (2013).

[5] A. A. Tsirlin, I. Rousochatzakis, D. Filimonov, D. Batuk, M. Frontzek, and A. M. Abakumov, Phys. Rev. B 96, 094420 (2017).

[6] J. Oh, M. D. Le, J. Jeong, J.-h. Lee, H. Woo, W.-Y. Song, T. G. Perring, W. J. L. Buyers, S.-W. Cheong, and J.-G. Park, Phys. Rev. Lett. 111, 257202 (2013).

[7] J. Oh, M. D. Le, H.-H. Nahm, H. Sim, J. Jeong, T. G. Perring, H. Woo, K. Nakajima, S. Ohira-Kawamura, Z. Yamani, Y. Yoshida, H. Eisaki, S.-W. Cheong, A. L. Chernyshev, and J.-G. Park, Nat. Commun. 7, 13146 (2016).

[8] U. Stuhr, B. Roessli, S. Gvasaliya, H. Rønnow, U. Filges, D. Graf, A. Bollhalder, D. Hohl, R. Bürge, M. Schild, L. Holitzner, C. Kaegi, P. Keller, and T. Mühlebach, Nucl. Instrum. Methods Phys. Res. A 853, 16 (2017).

[9] J. Kulda, J. Šaroun, P. Courtois, M. Enderle, M. Thomas, and P. Flores, Appl. Phys. A 74, s246 (2002).

[10] S. A. Kim, C.-H. Lee, M.-K. Moon, S.-J. Cho, T.-S. Yoon, Y. Noda, and Y. Ishikawa, Neutron News 24, 33 (2013).

[11] M. D. Le, M. Enderle, A. Tsirlin, and E. Wheeler, Determination of the exchange parameters in the frustrated cairo pentagon lattice compound $\mathrm{Bi}_{2} \mathrm{Fe}_{4} \mathrm{O}_{9}$, doi:10.5291/ILL-DATA.4-01-1428 (2015).

[12] M. D. Le, Magnetic excitations in a frustrated cairo pentagon lattice, doi:10.5286/ISIS.E.RB1820598 (2018).

[13] R. I. Bewley, T. Guidi, and S. Bennington, Notiziario Neutroni Luce Sincrotrone 14, 22 (2009).
[14] R. A. Ewings, J. R. Stewart, T. G. Perring, R. I. Bewley, M. D. Le, D. Raspino, D. E. Pooley, G. Škoro, S. P. Waller, D. Zacek, C. A. Smith, and R. C. Riehl-Shaw, Rev. Sci. Instrum. 90, 035110 (2019).

[15] M. D. Le, B. Fåk, A. Tsirlin, and E. Wheeler, Magnetic excitations in the frustrated Cairo pentagon lattice compound $\mathrm{Bi}_{4} \mathrm{Fe}_{5} \mathrm{O}_{13} \mathrm{~F}$, doi:10.5291/ILL-DATA.4-01-1427 (2015).

[16] G. Cicognani, H. Mutka, and F. Sacchetti, Phys. B: Condens. Matter 276-278, 83 (2000).

[17] D. Richard, M. Ferrand, and G. Kearley, J. Neutron Res. 4, 33 (1996).

[18] O. Arnold, J. Bilheux, J. Borreguero, A. Buts, S. Campbell, L. Chapon, M. Doucet, N. Draper, R. F. Leal, M. Gigg, V. Lynch, A. Markvardsen, D. Mikkelson, R. Mikkelson, R. Miller, K. Palmen, P. Parker, G. Passos, T. Perring, P. Peterson, S. Ren, M. Reuter, A. Savici, J. Taylor, R. Taylor, R. Tolchenov, W. Zhou, and J. Zikovsky, Nucl. Instrum. Methods Phys. Res. A 764, 156 (2014).

[19] S. Toth and B. Lake, J. Phys.: Condens. Matter 27, 166002 (2015).

[20] S. Tóth et al., http://www.spinw.org.

[21] M. Rotter, M. D. Le, A. T. Boothroyd, and J. A. Blanco, J. Phys.: Condens. Matter 24, 213201 (2012).

[22] M. Rotter et al., http://www.mcphase.de.

[23] K. Beauvois, V. Simonet, S. Petit, J. Robert, F. Bourdarot, M. Gospodinov, A. A. Mukhin, R. Ballou, V. Skumryev, and E. Ressouche, Phys. Rev. Lett. 124, 127202 (2020).

[24] M. Enderle, Collection SFN 13, 01002 (2014).

[25] Z. V. Pchelkina and S. V. Streltsov, Phys. Rev. B 88, 054424 (2013).

[26] In the literature for $\mathrm{Bi}_{2} \mathrm{Fe}_{4} \mathrm{O}_{9}, \mathrm{Fe}_{1}$ is used for the threefold sites we label $\mathrm{Fe}^{3}$ and $\mathrm{Fe}_{2}$ for the sites we label $\mathrm{Fe}^{4}[3,23]$. In contrast, the literature for $\mathrm{Bi}_{4} \mathrm{Fe}_{5} \mathrm{O}_{13} \mathrm{~F}$ uses $\mathrm{Fe}_{1}$ for the fourfold site $\mathrm{Fe}^{4}, \mathrm{Fe}_{2}$ for the threefold site $\mathrm{Fe}^{3}$, and $\mathrm{Fe}_{3}$ for the intermediate layer site $\mathrm{Fe}^{\text {int }}$ [5].

[27] M. D. Le et al., http://github.com/mducle/cairo-data. 